\title{
Comment on the paper by J. T. Jebsen reprinted in Gen. Rel. Grav. 37, 2253 - 2259 (2005)
}

\section{J. Ehlers • A. Krasiński}

Published online: 18 July 2006

(C) Springer-Verlag 2006

Jebsen [1] anticipated Birkhoff [2] in claiming that spherically symmetric vacuum space-times, possibly with $\Lambda$, are static. That statement is wrong, counterexamples are the extension of the Schwarzschild solution into the region behind the horizon and analogous parts of DeSitter and Anti-DS. The mistake in the proof occurs where Jebsen claims that in a spherically symmetric metric

$$
\mathrm{d} s^{2}=F(r, l) \mathrm{d} r^{2}+G(r, l)\left(\mathrm{d} \vartheta^{2}+\sin ^{2} \vartheta \mathrm{d} \varphi^{2}\right)+H(r, l) \mathrm{d} r \mathrm{~d} l+D(r, l) \mathrm{d} l^{2},
$$

by transforming the $(r, l)$ coordinates, one can achieve $H=0$ and $G=r^{2}$. $H=0$ can indeed be achieved, and in addition one can require $F>0, G>0$ and $D<0$. Then, if the gradient of $\sqrt{G}$ is spacelike (respectively timelike), one can put $G=r^{2}$ (resp. $G=l^{2}$ ). If $G$ is constant, no condition at all can be imposed on it; there exists an exact vacuum solution with $\Lambda>0$ in this class, found by Nariai [3]. If $\sqrt{G}$ has a light-like gradient, no vacuum solution for any $\Lambda$ exists.

In fact, all solutions of the required kind are known and can be continued to inextendable ones. A corrected version of the theorem states that all spherically symmetric solutions admit, besides the $\mathrm{SO}(3)$ generators, an additional hypersurface-orthogonal Killing vector field.

\footnotetext{
J. Ehlers · A. Krasiński $(\varangle)$

Polish Academy of Sciences, N. Copernicus Astronomical Center,

Bartycka 18, Warszawa 00 716, Poland

e-mail: akr@camk.edu.pl
} 


\section{References}

1. Jebsen, J.T.: Arkiv för Matematik. Astronomi och Fysik 15(18), 1-9 (1921)

2. Birkhoff, G.D.: Relativity and Modern Physics p. 256. Cambridge University Press Cambridge (1923)

3. Nariai, H.: Scientific reports of the Tôhoku University 34, 160 (1950); 35, 46 (1951); both papers reprinted in Gen. Rel. Grav. 31, 951 (1999) 